\section{Benchtop systems}

Advion Biosciences

Allegro Technologies

BD Biosciences

BioRobotics

Bio-Tek

BMG Labtechnologies

Boston Innovation

Colibri Robotics

Genevac

GenoVision

Gilson

IGEN International

MJ Research

OptiCell

Titertek

Torcon Instruments

Xiril

Zinsser Analytic
Fully automated nanoelectrospray for mass spectroscopy

Nanovolume pipetting systems

Multiwell AutoSampler for flow cytometers; high-throughput solubility scanner

MicroGrid automated arrayer and BioPick automated colony picker

Automated microplate handling and reading instrumentation, spectrophotometers

Microplate readers and handling systems

Automated sample delivery

Suppliers of Xiril pipetting robots and Plato automated microplate processors

Automated solvent removal systems

GenoM-6 automated system for DNA isolation and purification

Automated liquid-handling, pipetting and protein crystallization instruments

Automated assay analysers using electrochemiluminescence detection

DNA Engine thermal cyclers, 96- and 389-well hard-shell plates

Automated cell-culture devices

Precision liquid-handling and bench-top automation of microplate assays

Precision fluid dispensing and automated instrumentation for microplate handling

Pipetting robots

Pipetting, extraction and liquid-handling robots
Ithaca, New York

Dublin, Eire

Oxford, UK

Cambridge, UK

Winooski, Vermont

Offenburg, Germany

Cambridge, Massachusetts

New Castle, Delaware

Ipswich, UK

Westchester, Pennsylvania

Middleton, Wisconsin

Gaithersburg, Maryland

Waltham, Massachusetts

Westerville, Ohio

Huntsville, Alabama

Torrance, California

Hombrechtikon, Switzerland

Frankfurt, Germany www.advion.com

www.allegro-technologies.com

www.bdeurope.com

www.biorobotics.com

www.biotek.com

www.bmglabtech.com

www.bostoninnovation.com

www.colibrirobotics.com

www.genevac.com

www.genovision.com

www.gilson.com

www.igen.com/home.htm

www.mjr.com

www.opticell.com

www.titertek.com

www.torconinstruments.com

www.xiril.com

www.zinsser-analytic.com

\section{Microfluidics}

Aclara BioSciences

Caliper Technologies

Gyros

\section{Workstations}

Applied Biosystems

Affymetric

Agilent

Applied S

AutoGen

Beckman Coulter

Biotage

BioTec

Brandel

Bruker

Carl Zeiss

Cell Robotics Internationa

Chemical Diversity Labs

Cytogration

deCODE Genetics

Dynex Technologies

GeneMachines
Genetix
Genomic Solutions
Gentra Systems
Hamilton
Labcyte

Lab Services

Liconic Instruments

Magnetic Biosolutions

MWG-Biotech

Nanogen
QIAGEN
Relab
Sias
Tecan
Tomtec
Union Biometrica
Velocity11

Zymark
LabCard microfluidics arrays for high-throughput drug screening, multiplexed gene-expression analysis, and multiplexed SNP genotyping

LabChip microfluidic systems for high-throughput screening for drug discovery,

biological and genetic research

CD microlaboratory for sample preparation for protein mass spectrometry
Mountain View, California

Mountain View, California

Uppsala, Sweden

www.calipertech.com

www.gyros.com

\section{www.aclara.com}

Foster City, California Santa Clara, California DNA sequencers, mass spectrometers, workstations and automated instruments GeneChip microarray systems

Workstation for RNA, DNA or protein analysis, instruments for genomics and proteomics Palo Alto, California
Automated systems for microscopy and fluorescence screening Automated nucleic acid extraction instruments and colony/plaque-picking systems Automation tools from modular liquid-handling systems to integrated robotic systems Horizon range of high-performance FLASH chromatography systems Liquid-handling lab instruments, pipetting workstation, dispenser, microplate washer Automated sample preparation and harvesting, superfusion systems, microdispensers Automated platforms for mass spectroscopy, NMR, EPR and magnetic resonance plate:::explorer and plate:::screen automation for ultra-high throughput screening Microscope workstations for biomedical research CombiSyn reactors for solid- and solution-phase parallel synthesis Automated high-throughput cell culture systems for in vitro screening Emerald Biostructures products and robotic platform for protein crystallization Absorbance and luminescence microplate readers, microplate washers and automated workstations

Automated instruments for sample preparation, microarraying, DNA purification, oligonucleotide synthesis and colony/plaque picking

Picking, arraying, gridding, replicating, rearraying and microarraying robots Automated instrumentation, software, consumables and services

Automated equipment for nucleic acid purification Microlab automated liquid-handling workstations

Automated pipetting workstations, microplate washers, magnetic-bead washers and DNA-extraction working decks

Automated liquid-handling instruments, fluorescence readers and microplate labs Lab automation, automated incubators and storage systems Robotic workstations for automated biomagnetic separation Thermal cyclers, automated systems for high-throughput sequencing and microarray production NanoChip automated workstation for SNP and STR analysis BioRobot workstation; automated nucleic acid, protein and cell purification Arrays, scanners, workstations and dispensers for biochips Xantus robotic liquid-handling platform; Ixion robot-friendly microplate centrifuge Laboratory automation for the life sciences, LabCD 'lab-on-a-disc' assays Liquid-handling and solid-phase extraction workstations; automated instruments COPAS platforms for automated high-throughput analysis, sorting and dispensing of small multicellular organisms

BioCel automation platform for high-throughput microplate processing, microplate dispensers and pipetting stations Sciclone liquid-handling stations, Staccato high-throughput workstations
Holliston, Massachusetts

Fullerton, California

Charlottesville, Virginia

Tokyo Japan

Gaithersburg, Maryland

The Woodlands, Texas

Jena, Germany

Albuquerque, New Mexico

San Diego, California

Rockville, Maryland

Bainbridge Island, Washington

Chantilly, Virginia

San Carlos, California

New Milton, UK

Ann Arbor, Michigan

Minneapolis, Minnesota

Bonaduz, Switzerland

Union City, California

Breda, the Netherlands Mauren, Liechtenstein

Stockholm, Sweden

Ebersberg, Germany

San Diego, California

Venlo, The Netherlands

Recklinghausen, Germany

Hombrechtikon, Switzerland

Männedorf, Switzerland

Hamden, Connecticut

Somerville, Massachusetts

Palo Alto, California

Hopkinton, Massachusetts home.appliedbiosystems.com

www.affymetrix.com

www.agilent.com

www.asiimaging.com

www.autogen.com

www.beckman.com

www.biotage.com

www.biotec.co.jp

www.brandel.com

www.bruker.com

www.zeiss.de

www.cellrobotics.com

www.chemdiv.com

www.cytogration.com

www.decode.com

www.dynextechnologies.com

www.genemachines.com

www.genetix.co.uk

www.genomicsolutions.com

www.gentra.com

www.hamiltoncomp.com

www.labcyte.com

www.lab-services.n

www.liconic.com

www.magbio.com

www.mwg-biotech.com

www.nanogen.com

www.qiagen.com

www.relab.de

www.sias.biz

www.tecan.com

www.tomtec.com

www unionbio.com

www.velocity11.com 


\section{Integrated laboratory automation}

AB Controls

accelab

Biometra

Cellomics

CyBio

DataCentric Automation

$\mathrm{H}+\mathrm{P}$ Labortechnik

Hettich-Zentrifugen

Hitachi High-Technologies

IBM Corporation Life Sciences

J-KEM Scientific

KBiosystems

Kendro Laboratory Products

Molecular Devices

Custom hardware and software for laboratory automation and robotics

Customized laboratory automation

TRobot thermocycler for integration with robotic environments

ArrayScan HCS and KineticScan HCS for automated cell-based screening

Pipetting, liquid handling, incubation, and imaging systems for automated screening

Automated systems for protein crystallography

VARIOMAG magnetic stirrers, shakers and reaction blocks

ROTANTA 46 RSC robotic centrifuge

Automated instrumentation for mass spectroscopy, liquid chromatography,

high-throughput purification, amino-acid analysis and spectroscopy

DiscoveryLink platform for database integration; data-management systems

Customized automated laboratory equipment

Robotic instrumentation for high-throughput genomics and proteomics

Cytomat robot-accessible incubators

Liquid-handling and microplate-processing; imaging; assay systems

Process Analysis and Automation Automated laboratory instruments and software

Protedyne

RTS Life Sciences

BioCube automated systems; LIMS middleware and integration software

Automation for sample management, liquid handling, ultra-high-throughput screening and cell culture, and LIMS

Seyonic

Synbiosis

Syncroscopy

Syngene

The Automation Partnership

Nanolitre sensor-controlled liquid-handling systems; microarray spotting systems

Microbiology equipment to automate microbial colony counting and zone sizing

Digital imaging systems for microscopy

Systems for documenting and analysing gels

Thermo CRS

Automation for liquid handling, compound storage and retrieval, high-throughput

screening, genomics and robotic cell culture

Integrated laboratory automation, magnetic bead-based separation technology
Irvine, California

Kusterdingen, Germany

Göttingen, Germany

Pittsburgh, Pennsylvania

Jena, Germany

Nashville, Tennessee

Oberschleißheim, Germany

Tuttlingen, Germany

Tokyo, Japan

Somers, New York

St Louis, Missouri

Basildon, UK

Hanau, Germany

Sunnyvale, California

Farnborough, UK

Windsor, Connecticut

Manchester, UK

Neuchåtel, Switzerland

Cambridge, UK

Cambridge, UK

Cambridge, UK

Royston, UK

Burlington, Ontario

Piscataway, New Jersey

Columbia, Maryland

Jena, Germany

Menlo Park, California

Guelph, Ontario, Canada

High Wycombe, UK

South Hollywood, Florida

Tokyo, Japan

Earth City, Missouri

Tucson, Arizona

Manchester, UK

Windows-based LIMS for HLA typing

LIMS, chromatography data-archiving and spectroscopy software

Sunquest HLA

Thermo Labsystems

Customized LIMS

StarLIMS laboratory information management system

LIMS with barcode

d retrieval systems

Biophile

Ultra-low-temperature sample storage and retrieval system

TekCe

Automated tube and microplate management, low-temperature storage/retrieval

TTP LabTech

Universal Technology

Automated storage/retrieval, nanolitre liquid-handling, fluorescence detection

Automated storage and retrieval systems for the pharmaceutical industry

Charlottesville, Virginia

Hopkinton, Massachusetts

Royston, UK

Pittsburgh, Pennsylvania www.abcontrols.com

www.accelab.de

www.biometra.de

www.cellomics.com

www.cybio-ag.com

www.titanceg.com/divisions

www.hp-lab.de

www.hettichlab.com

www.hitachi-hitec.com

www.ibm.com/solutions/lifesciences

www.jkem.com/index.htm

www.kbiosystems.com

www.kendro.com

www.moleculardevices.com

www.paa.co.uk

www.protedyne.com

www.rts-group.com

www.seyonic.com

www.synbiosis.com

www.syncroscopy.com

www.syngene.com

www.automationpartnership.com

www.crsrobotics.com

\section{General}

ACME-Automation

Bio-Automation

Brinkmann

BSI Proteomics

Compugen

Hudson Control Group

Microbiology International

Millipore

Nunc

PerkinElmer Life Sciences

Phenix1

Pierce Chemical

RoboDesign

Rohasys

Sankyo Robotics

Structural GenomiX

Syrrx

Treff

Whatman

- See advertisement
Services and products for laboratory automation

Bio-Bot linear-track robotic arm

Laboratory instrument suppliers

Proprietary system for automated protein crystallization for crystallography

Z4000 analysis system for complex proteomics

Modular instrumentation for use in automated systems

Automated equipment for microbiology

Automated equipment for molecular biology, biochemistry, genomics and proteomics

Microtitre plates

Automated systems for liquid handling and sample preparation

Suppliers of automated equipment and consumables for molecular biology

Components and consumables for automated systems in protein chemistry, sample handling, immunology and chromatography

Automated equipment for biotech, protein structure, genomics and proteomics

Automated equipment for pretreatment, weighing, $\mathrm{pH}$, conductivity, turbidity

Multi-purpose robots

Automated protein crystallography for drug discovery

Automated protein crystallography for drug discovery

Customized plastic consumables including deep-well and PCR plates

Separation technology, microplates, Biometra instruments, and components
Spring City, Tennessee Cheshire, Connecticut

Westbury, New York

Gaithersburg, Maryland

Tel Aviv, Israel

Springfield, New Jersey

Frederick, Maryland

Bedford, Massachusetts

Roskilde, Denmark

Boston, Massachusetts

Hayward, California

Rockford, Illinois

Carlsbad, California

Rijen, the Netherlands

Boca Raton, Florida

San Diego, California

San Diego, California

Degersheim, Switzerland

Maidstone, UK www.amershambiosciences.com

www.avantium.com

www.clondiag.com

www.geneticxchange.com

www.labtronics.com

www.labvantage.com

www.starlims.com

bio.mki.co.jp

www.scinomix.com

www.sunquest.com

www.thermolabsystems.com www.biophileinc.com www.tekcel.com www.ttplabtech.com www.univtech.com 